PROCEEDINGS OF THE

AMERICAN MATHEMATICAL SOCIETY

Volume 137, Number 8, August 2009, Pages 2671-2683

S 0002-9939(09)09803-7

Article electronically published on February 3, 2009

\title{
SUR L'EXISTENCE D'UNE SOLUTION RAMIFIÉE POUR DES ÉQUATIONS DE FUCHS À CARACTÉRISTIQUE SIMPLE
}

\author{
PATRICE PONGÉRARD
}

(Communicated by Matthew J. Gursky)

\begin{abstract}
The aim of this paper is to construct a holomorphic solution, ramified around a simple characteristic hypersurface, for some linear Fuchsian equation of order $m \geq 1$. We consider an operator $L$, holomorphic in a neighborhood of the origin in $\mathbb{C}_{t} \times \mathbb{C}_{x}^{n}$, of the form $L=t A+B$ where $A$ and $B$ are linear partial differential operators of order $m$ and $m-1$, and where $A$ has a simple characteristic hypersurface transverse to $S: t=0$. Under an assumption linking the principal symbols of $A$ and $B$, the question is reduced to the study of an integro-differential Fuchsian equation with an additional variable $z$ that describes the universal covering of a pointed disk. It is an equation where terms like $t^{l} D_{t}^{h} D_{x}^{\alpha}\left(t D_{t}+1\right)^{-1} D_{z}^{-q}, l, h, q \in \mathbb{N}, \alpha \in \mathbb{N}^{n}$ with $l \leq 1$ and $h+|\alpha| \leq l+q$ appear. The problem is solved by the fixed-point theorem with appropriate estimations in a Banach space.
\end{abstract}

\section{Notations et RÉSultat}

Les coordonnées d'un point $(t, x)$ de $\mathbb{C} \times \mathbb{C}^{n}$ seront notées $\left(t, x_{1}, \ldots, x_{n}\right)$. L'ensemble des entiers naturels étant noté $\mathbb{N}=\{0,1,2, \ldots\}$, l'opérateur de dérivation par rapport à la variable $t\left(\right.$ resp. $\left.x_{j}\right)$ sera noté $D_{t}\left(\right.$ resp. $\left.D_{j}\right)$ et nous poserons $D_{t}^{l}$ et $D^{\alpha}=D_{1}^{\alpha_{1}} \cdots D_{n}^{\alpha_{n}}$ pour tout $l \in \mathbb{N}$ et tout $\alpha=\left(\alpha_{1}, \ldots, \alpha_{n}\right) \in \mathbb{N}^{n}$. On considère un opérateur différentiel linéaire $L$, à coefficients holomorphes au voisinage de l'origine de $\mathbb{C} \times \mathbb{C}^{n}$, de la forme

$$
L\left(t, x ; D_{t}, D\right)=t A\left(t, x ; D_{t}, D\right)+B\left(t, x ; D_{t}, D\right),
$$

où $A$ (resp. $B$ ) est un opérateur différentiel linéaire d'ordre $m \geq 1$ (resp. $m-1$ ) dont le symbole principal sera noté $g_{A}\left(\right.$ resp. $\left.g_{B}\right)$ et où $g_{A}(\bullet ; 1,0) \equiv 1$. On vérifie que $L$ est un opérateur différentiel fuchsien d'ordre $m$ et de poids $m-1$ ayant pour partie fuchsienne

$$
a\left(t, x, D_{t}\right) \equiv g_{A}(0, x ; 1,0) t D_{t}^{m}+g_{B}(0, x ; 1,0) D_{t}^{m-1} .
$$

On note $\mathcal{O}_{0}=\mathcal{D}_{0} \times \Omega_{0}$ un voisinage ouvert de l'origine de $\mathbb{C} \times \mathbb{C}^{n}$ sur lequel tous les coefficients de l'opérateur $L$ sont définis et holomorphes.

Received by the editors February 25, 2008, and, in revised form, October 16, 2008

2000 Mathematics Subject Classification. Primary 35A07; Secondary 35A20.

(C)2009 American Mathematical Society 
Nous supposons que le polynôme $\tau \mapsto g_{A}(0 ; \tau, 1,0, \ldots, 0)$ admet une racine simple $\bar{\tau}$. On a donc

$$
D_{\tau} g_{A}(0 ; \bar{\tau}, 1,0, \ldots, 0) \neq 0
$$

ce qui assure que le problème

$$
\begin{cases}g_{A}(t, x ; \nabla k(t, x)) & =0, \\ \nabla k(0) & =(\bar{\tau}, 1,0, \ldots, 0), \\ k(0, x) & =x_{1}\end{cases}
$$

admet une unique solution holomorphe au voisinage de l'origine de $\mathbb{C} \times \mathbb{C}^{n}$; on peut donc supposer que la fonction $k$ est définie et holomorphe sur $\mathcal{O}_{0}$ et que $D_{1} k(t, x) \neq 0$ pour tout $(t, x) \in \mathcal{O}_{0}$. Ceci permet de définir une hypersurface $K=\left\{(t, x) \in \mathcal{O}_{0} \mid k(t, x)=0\right\}$ transverse à $S$. En notant $T$ l'hyperplan de $S$ d'équation $t=x_{1}=0$, on a $K \cap S=\Omega_{0} \cap T$ : l'ensemble $K$ est une hypersurface caractéristique simple issue de $T$ et transverse à $S$.

Pour tout $\delta>0$, on pose $D_{\delta}=\{z \in \mathbb{C}|| z \mid<\delta\}$ et on note $\mathcal{R}_{\delta}$ le revêtement universel du disque pointé $\dot{D}_{\delta}=\{z \in \mathbb{C}|0<| z \mid<\delta\}$. Si $v$ est une fonction holomorphe ramifiée autour de $K$, on peut trouver un réel $\delta>0$ et un voisinage ouvert connexe $\mathcal{O} \subset \mathcal{O}_{0}$ de l'origine de $\mathbb{C} \times \mathbb{C}^{n}$ tels que $v$ soit de la forme

$$
v(t, x)=\tilde{v}(z, t, x)_{\mid z=k(t, x)},
$$

où $\tilde{v}$ est une fonction holomorphe sur $\mathcal{R}_{\delta} \times \mathcal{O}$; quitte à réduire $\mathcal{O}$, on peut supposer que $|k(t, x)|<\delta$ pour tout $(t, x) \in \mathcal{O}$. La fonction (1.5) est alors définie et holomorphe sur le revêtement universel de $\mathcal{O}-K$.

On se propose de construire une solution de l'équation

$$
L\left(t, x ; D_{t}, D\right) u(t, x)=\left.v(z, t, x)\right|_{z=k(t, x)} .
$$

Cette construction est possible si l'opérateur $L$ satisfait à la condition suivante. On utilisera la partie fuchsienne d'ordre 1 et de poids 0 ,

$$
b\left(t, x, D_{t}\right) \equiv D_{\tau} g_{A}(0, x ; \nabla k(0, x)) t D_{t}+g_{B}(0, x ; \nabla k(0, x)),
$$

à coefficients définis et holomorphes sur $\Omega_{0}$. On associe à $b$ son polynôme caractéristique

$$
P(x, \lambda)=D_{\tau} g_{A}(0, x ; \nabla k(0, x)) \lambda+g_{B}(0, x ; \nabla k(0, x)),
$$

qui vérifie $P\left(x, t D_{t}\right)=b\left(t, x, D_{t}\right)$, et on suppose que

$$
P(0, \lambda) \neq 0 \text { pour tout } \lambda \in \mathbb{N} \text {. }
$$

On a alors le théorème suivant.

Théorème 1.1. Soit $\mathcal{O} \subset \mathcal{O}_{0}$ un voisinage ouvert de l'origine de $\mathbb{C} \times \mathbb{C}^{n}$, il existe $\delta_{0}>0$ et un voisinage ouvert $\mathcal{O}^{\prime} \subset \mathcal{O}$ de l'origine de $\mathbb{C} \times \mathbb{C}^{n}$ tels que : soit $0<\delta \leq \delta_{0}$, pour toute fonction $v \in \mathcal{H}\left(\mathcal{R}_{\delta} \times \mathcal{O}\right)$, il existe une solution de l'équation (1.6) de la forme $u(k(t, x), t, x)$, où $u \in \mathcal{H}\left(\mathcal{R}_{\delta} \times \mathcal{O}^{\prime}\right)$.

Remarque 1.2. Considérons le problème (2.6)-(2.7) de [6] :

$$
\begin{cases}A\left(t, x ; D_{t}, D\right)\left[\left.u(z, t, x)\right|_{z=k(t, x)}\right] & =w_{1}(k(t, x), t, x), \\ u(z, t, x)-w_{0}(z, t, x) & =0 \text { pour } t=0\end{cases}
$$


avec des fonctions $w_{0}, w_{1}$ qui sont holomorphes au voisinage de $\mathcal{R}_{\delta} \times\{0\}$ pour un $\delta>0$. En prenant comme inconnue $w_{0}+t u$, le problème s'écrit

$$
\left(t A+D_{\tau} A\right)\left[\left.u(z, t, x)\right|_{z=k(t, x)}\right]=w_{1}-A\left[\left.w_{0}(z, t, x)\right|_{z=k(t, x)}\right] .
$$

Pour cette équation, la condition (1.8) s'écrit simplement

$$
D_{\tau} g_{A}(0 ; \nabla k(0))(\lambda+1) \neq 0 \quad \text { lorsque } \lambda \in \mathbb{N},
$$

soit $D_{\tau} g_{A}(0 ; \nabla k(0)) \neq 0$; autrement dit, la condition (1.8) est vide dans ce cas. Ceci prouve que le problème (1.9) est un cas particulier du problème (1.6)-(1.8).

Remarque 1.3. Il n'y a en général aucune relation entre (1.2) et (1.7) sauf lorsque $m=1$, auquel cas, on a $a\left(t, x, D_{t}\right)=b\left(t, x, D_{t}\right)$ et l'équation (1.6) est une équation fuchsienne de poids 0 : le théorème 1.1 donne l'existence d'une solution; on a également l'unicité dans ce cas d'après (1.8) et le lemme 1.4 de [11. Considérons ensuite le problème de Cauchy

$$
\begin{cases}L\left(t, x ; D_{t}, D\right) u(t, x) & =v(t, x), \\ D_{t}^{h} u(0, x) & =w_{h}(x) \text { pour } 0 \leq h<m-1\end{cases}
$$

et notons $\mathcal{C}(x, \lambda)$ le polynôme caractéristique de la partie fuchsienne $a\left(t, x ; D_{t}, D\right)$. Sous la condition $\mathcal{C}(0, k) \neq 0$ pour tout entier $k \geq m-1$, nous savons (BaouendiGoulaouic, 1]) que pour toutes fonctions $\left(w_{h}\right)_{0 \leq h<m-1}$ et $v$ holomorphes au voisinage de $x=0$ et $(t, x)=(0,0)$ respectivement, le problème de Cauchy (1.10) admet une unique solution holomorphe au voisinage de $(t, x)=(0,0)$.

Supposons que l'opérateur $L$ soit d'ordre 2, qu'il admette deux hypersurfaces caractéristiques simples $K_{1}, K_{2}$ transverses à $S$ et issues de $T$ et que l'ensemble $V=\bigcup_{\lambda \in \mathbb{N}^{*}}\left\{x \in \Omega_{0} \mid \mathcal{C}(x, \lambda)=0\right\}$ ait pour équation locale, $V: x_{1}=0$. Lorsque $w_{0}$ et $v$ sont des fonctions holomorphes ramifiées autour de $T$ et de $K_{1}$ respectivement, S. Ouchi a montré [7, Théorèmes 1.7 et 1.8] que toute solution de (1.10) (holomorphe au voisinage d'un point $(0, \alpha) \in S, \alpha \notin V)$ se prolonge en une fonction holomorphe ramifiée autour de $S \cup K_{1} \cup K_{2}$.

Nous étudions ici des opérateurs d'ordre $m \geq 1$ et aucune hypothèse n'est faite sur la partie fuchsienne $a\left(t, x ; D_{t}, D\right)$; sous la condition (1.8) qui porte sur l'opérateur $b\left(t, x ; D_{t}, D\right)$, nous obtenons le théorème 1.1.

Exemple 1.4. Considérons au voisinage de l'origine de $\mathbb{C}_{t} \times \mathbb{C}_{x}$ le cas d'un opérateur d'ordre 2 de la forme $L=t A+B$, où

$$
A \equiv D_{t}^{2}-D_{x}^{2} \quad \text { et } \quad B \equiv a(x) D_{t} .
$$

La partie fuchsienne de $L$ s'écrit $t D_{t}^{2}+a(x) D_{t}$ et a pour polynôme caractéristique

$$
\mathcal{C}(x, \lambda)=\lambda[\lambda+a(x)-1] .
$$

La condition $\mathcal{C}(0, k) \neq 0$ pour tout entier $k \geq 1$ signifie $a(0) \notin-\mathbb{N}$. Si $a(0) \in$ $-\mathbb{N}$, nous avons montré [11] que toute solution du problème de Cauchy (1.10) (holomorphe au voisinage d'un point $(0, \alpha) \in S, \alpha \neq 0)$ se prolonge en une fonction holomorphe sur le revêtement universel d'un ouvert de la forme $\{(t, x) \in \mathbb{C} \times \Omega \mid$ $\left.|t| \leq \kappa|x|^{2}\right\}$. Pour un $a$ particulier tel que $a(0) \in-\mathbb{N}$ est impair et $a \not \equiv 0$, S. Fujiié [2] a montré que le problème de Cauchy (1.10) admet une unique solution (holomorphe au voisinage d'un point $(0, \alpha) \in S, \alpha \neq 0$ ) qui se prolonge en une fonction holomorphe ramifiée autour de $S \cup K_{1} \cup K_{2}$. Revenons à la condition 
(1.8) ; le polynôme $g_{A}(\tau, 1)=\tau^{2}-1$ admet deux racines distinctes $\tau_{1}=1=-\tau_{2}$ et on a $K_{i}: \tau_{i} t+x=0, i=1,2$. La condition (1.8) s'écrit : pour tout $\lambda \in \mathbb{N}$,

$$
D_{\tau} g_{A}\left(\nabla k_{i}\right) \lambda+a(0) \tau_{i} \neq 0, \quad(i=1 \text { ou } 2)
$$

soit $2 \lambda+a(0) \neq 0$, autrement dit $a(0) \in-\mathbb{N}$ est impair. Dans ce cas, si $v$ est ramifiée autour de $K_{i}$, il existe d'après le théorème 1.1 une solution de l'équation $L u=v$, ramifiée autour de $K_{i}$.

\section{RÉDUCTION}

On cherche a priori une solution sous la forme $\left.u(z, t, x)\right|_{z=k(t, x)}$ et, pour expliciter (1.6), c'est-à-dire

$$
L\left(t, x ; D_{t}, D\right)\left[\left.u(z, t, x)\right|_{z=k(t, x)}\right]=v(z, t, x)_{\mid z=k(t, x)},
$$

on utilise le lemme suivant [8, Lemme 6.1].

Lemme 2.1. Soient $M\left(t, x ; D_{t}, D\right)$ un opérateur différentiel linéaire d'ordre $m$ à coefficients holomorphes dans un ouvert $\mathcal{O}$ de $\mathbb{C} \times \mathbb{C}^{n}$ et $k: \mathcal{O} \mapsto \mathbb{C}$ une fonction holomorphe. Il existe des opérateurs différentiels linéaires $M_{q}\left(t, x ; D_{t}, D\right), 0 \leq q \leq$ $m$, d'ordre $\leq q$ à coefficients holomorphes dans $\mathcal{O}$ tels que, pour tout a $\in \mathcal{O}$ et tout germe $u$ au point $(k(a), a) \in \mathbb{C} \times \mathcal{O}$, on ait pour $(t, x)$ voisin de $a$,

$$
M\left(t, x ; D_{t}, D\right) u(k(t, x), t, x)=\left.\sum_{q=0}^{m} M_{q}\left(t, x ; D_{t}, D\right) D_{z}^{m-q} u(z, t, x)\right|_{z=k(t, x)} .
$$

En outre, les coefficients de $M_{q}$ sont des combinaisons linéaires de ceux de $M$ dont les coefficients sont des polynômes en les dérivées de $k$. Si g est le symbole principal de $M$, le symbole principal de $M_{q}$, en tant qu'opérateur d'orde q, est donné par la formule

$$
\sigma\left(M_{q}\right)(t, x ; \tau, \xi)=\sum_{h+|\alpha|=q} D_{\tau}^{h} D_{\xi}^{\alpha} g(t, x ; \nabla k(t, x)) \frac{\tau^{h} \xi^{\alpha}}{h ! \alpha !} .
$$

D'après ce lemme, on a

$$
\left\{\begin{array}{l}
A[u(k(t, x), t, x)]=\left.\sum_{q=0}^{m} A_{q}\left(t, x ; D_{t}, D\right) D_{z}^{m-q} u(z, t, x)\right|_{z=k(t, x)}, \\
B[u(k(t, x), t, x)]=\left.\sum_{q=0}^{m-1} B_{q}\left(t, x ; D_{t}, D\right) D_{z}^{m-1-q} u(z, t, x)\right|_{z=k(t, x)},
\end{array}\right.
$$

où les opérateurs $A_{q}$ et $B_{q}$ sont d'ordre $\leq q$; vu (1.4) et (2.1), on constate que $A_{0}=\sigma\left(A_{0}\right)=0$ et, par conséquent,

$$
L\left(t, x ; D_{t}, D\right) u(k(t, x), t, x)=\left.\sum_{q=0}^{m-1}\left(t A_{q+1}+B_{q}\right) D_{z}^{m-1-q} u(z, t, x)\right|_{z=k(t, x)} .
$$

Introduisons maintenant un inverse à droite de l'opérateur de dérivation $D_{z}$. Soient $\mathcal{R}_{\delta}$ le revêtement universel du disque pointé $\dot{D}_{\delta}$ et $\pi: \mathcal{R}_{\delta} \rightarrow \dot{D}_{\delta}$ la surjection canonique. On choisit un point $\hat{a}_{1}$ de $\mathcal{R}_{\delta}$ tel que $\pi\left(\hat{a}_{1}\right)=a_{1}$ et pour toute fonction holomorphe $u: \mathcal{R}_{\delta} \times \mathcal{O} \rightarrow \mathbb{C}$, on définit la primitive de $u$ qui s'annule au point $\hat{a}_{1}$ par

$$
D_{z}^{-1} u(z, t, x)=\int_{\hat{a}_{1}}^{z} u(\sigma, t, x) d \sigma,
$$

où l'intégrale s'effectue sur des chemins joignant les points $\hat{a}_{1}$ et $z$; bien entendu, la valeur de cette intégrale ne dépend pas du chemin choisi. On obtient ainsi une 
fonction holomorphe $D_{z}^{-1} u: \mathcal{R}_{\delta} \times \mathcal{O} \rightarrow \mathbb{C}$ et un opérateur $D_{z}^{-1}: \mathcal{H}\left(\mathcal{R}_{\delta} \times \mathcal{O}\right) \rightarrow$ $\mathcal{H}\left(\mathcal{R}_{\delta} \times \mathcal{O}\right)$ vérifiant $D_{z} \circ D_{z}^{-1}=I d$.

Lemme 2.2. Soient $\delta>0$ et $\mathcal{O}$ un ouvert de $\mathbb{C} \times \mathbb{C}^{n}$. L'opérateur $D_{z}^{-1}$ est un endomorphisme continu de l'espace $\mathcal{H}\left(\mathcal{R}_{\delta} \times \mathcal{O}\right)$.

Démonstration. Considérons le revêtement universel de $\dot{D}_{\delta}$ défini par l'ouvert simplement connexe $Z_{\delta}=\left\{\zeta \in \mathbb{C}^{\star} \mid e^{\zeta} \in \dot{D}_{\delta}\right\}$ et par l'application $p: Z_{\delta} \rightarrow \dot{D}_{\delta}$, où $p(\zeta)=e^{\zeta}$. Il existe un homéomorphisme $h: \mathcal{R}_{\delta} \rightarrow Z_{\delta}$ tel que $\pi=p \circ h$. Soit $K$ une partie compacte de $\mathcal{R}_{\delta}$, posons

$$
K^{\prime}=\bigcup_{z \in K} h^{-1}\left(\left[h\left(\hat{a}_{1}\right), h(z)\right]\right) .
$$

Il est clair que $K^{\prime}=h^{-1}(L)$, où $L=\bigcup_{\zeta \in h(K)}\left[h\left(\hat{a}_{1}\right), \zeta\right]$ est une partie compacte de $Z_{\delta}$. Soit $\gamma:[0,1] \rightarrow Z_{\delta}$ le segment joignant $h\left(\hat{a}_{1}\right)$ et $h(z)$, on a

$$
D_{z}^{-1} u(z, t, x)=\int_{\hat{a}_{1}}^{z} u(\sigma, t, x) d \sigma=\int_{0}^{1} u\left(h^{-1} \circ \gamma(s), t, x\right)(p \circ \gamma)^{\prime}(s) d s
$$

car $\pi \circ h^{-1}=p$. Si $M$ est une partie compacte de $\mathcal{O}$, on obtien finalement

$$
\max _{K \times M}\left|D_{z}^{-1} u\right| \leq c \max _{K^{\prime} \times M}|u|, \quad \text { où } \quad c \equiv \max _{K}\left|h-h\left(\hat{a}_{1}\right)\right| \times \max _{L}|p|,
$$

d'où le lemme.

On peut ensuite écrire

$$
L\left(t, x ; D_{t}, D\right) u(k(t, x), t, x)=\left.D_{z}^{m-1} \sum_{q=0}^{m-1}\left(t A_{q+1}+B_{q}\right) D_{z}^{-q} u(z, t, x)\right|_{z=k(t, x)} .
$$

En outre, d'après $(2.1)$, on a

$$
B_{0}(t, x)=g_{B}(t, x ; \nabla k(t, x))
$$

et

$$
\sigma\left(A_{1}\right)(t, x ; \tau, \xi)=D_{\tau} g_{A}(t, x ; \nabla k(t, x)) \tau+\sum_{j=1}^{n} D_{\xi_{j}} g_{A}(t, x ; \nabla k(t, x)) \xi_{j} .
$$

En utilisant la notation (1.7), il en résulte que

$$
\left\{\left(t A_{1}+B_{0}\right)\left(t, x ; D_{t}, D\right)=b\left(t, x ; D_{t}\right)+\sum_{l+|\alpha| \leq 1} a_{l, \alpha}(t, x) t^{l+1} D_{t}^{l} D^{\alpha},\right.
$$

où les $a_{l, \alpha}$ sont des fonctions holomorphes sur $\mathcal{O}_{0}$. En notant, pour $1 \leq q \leq m-1$,

$$
A_{q}^{l+q}= \begin{cases}B_{q} & \text { si } l=0, \\ A_{q+1} & \text { si } l=1,\end{cases}
$$

on obtient finalement

$$
\begin{aligned}
L\left(t, x ; D_{t}, D\right) u(k(t, x), t, x) & =D_{z}^{m-1}\left(b\left(t, x ; D_{t}\right)+\sum_{l+|\alpha| \leq 1} a_{l, \alpha}(t, x) t^{1+l} D_{t}^{l} D^{\alpha}\right. \\
& \left.+\sum_{\substack{0 \leq l \leq 1 \\
1 \leq q \leq m-1}} t^{l} A_{q}^{l+q}\left(t, x ; D_{t}, D\right) D_{z}^{-q}\right)\left.u(z, t, x)\right|_{z=k(t, x)},
\end{aligned}
$$


où l'ordre des opérateurs $A_{q}^{l+q}$ est $\leq l+q$. Notons que $k(0, a)=a_{1}$ d'après (1.4); pour que (1.6) soit vérifé, il suffit d'avoir, pour $(z, t, x)$ voisin de $\left(a_{1}, 0, a\right)$,

$$
\left(b\left(t, x ; D_{t}\right)+\sum_{l+|\alpha| \leq 1} a_{l, \alpha}(t, x) t^{1+l} D_{t}^{l} D^{\alpha}+\sum_{\substack{0 \leq l \leq 1 \\ 1 \leq q \leq m-1}} t^{l} A_{q}^{l+q}\left(t, x ; D_{t}, D\right) D_{z}^{-q}\right) u=D_{z}^{1-m} v .
$$

Autrement dit, il suffit d'étudier une équation de la forme

$$
\begin{aligned}
P\left(x, t D_{t}\right) u=\sum_{l+|\alpha| \leq 1} a_{l, \alpha}(t, x) t^{1+l} D_{t}^{l} D^{\alpha} & \\
& +\sum_{\substack{0 \leq l \leq 1 \\
1 \leq q \leq m-1}} t^{l} A_{q}^{l+q}\left(t, x ; D_{t}, D\right) D_{z}^{-q} u+v,
\end{aligned}
$$

où $P$ demeure inchangé et, après avoir changé de notations, les $a_{l, \alpha}$ sont des fonctions holomorphes sur $\mathcal{O}_{0}$, les $A_{q}^{l+q}$ sont des opérateurs différentiels linéaires holomorphes sur $\mathcal{O}_{0}$ d'ordre $\leq l+q$ et $v$ est une fonction appartenant à l'espace $\mathcal{H}\left(\mathcal{R}_{\delta} \times \mathcal{O}\right)$. Il s'agit d'une équation fuchsienne intégro-différentielle dont l'étude ne figure pas, semble-t-il, dans les travaux antérieurs.

L'hypothèse (1.8) permet d'établir le lemme suivant. Pour tout $R>0$, on pose

$$
D_{R}=\{t \in \mathbb{C}|| t \mid<R\} .
$$

Lemme 2.3. Il existe un voisinage ouvert $\Omega_{1} \subset \Omega_{0}$ de l'origine de $\mathbb{C}^{n}$ et une constante $c_{0}>0$ tels que :

a. $|P(x, k)| \geq c_{0}(k+1)$ pour tout $x \in \Omega_{1}$ et tout $k \in \mathbb{N}$.

b. Soient $\delta>0, R>0$ et $\Omega \subset \Omega_{1}$ un ouvert de $\mathbb{C}^{n}$. L'opérateur $P \equiv P\left(x, t D_{t}\right)$ est un homéomorphisme linéaire de l'espace $\mathcal{H}\left(\mathcal{R}_{\delta} \times D_{R} \times \Omega\right)$ sur lui même et

$$
\left(P^{-1} u\right)(z, t, x)=\sum_{k \in \mathbb{N}} \frac{t^{k}}{k !} \frac{D_{t}^{k} u(z, 0, x)}{P(x, k)} ;
$$

de plus, les opérateurs $P^{-1}$ et $D_{z}^{q}$ commutent quel que soit $q \in \mathbb{Z}$.

c. Soient $\delta>0, R^{\prime}>0$ et $\mathcal{U}$ une partie de l'ensemble des ouverts de $\Omega_{1}$. L'assertion $b$ est encore vraie lorsque l'ouvert $D_{R} \times \Omega$ est remplacé par un ouvert de la forme

$$
\mathcal{O}=\bigcup_{(R, \Omega) \in] 0, R^{\prime}[\times \mathcal{U}} D_{R} \times \Omega
$$

Démonstration. a. On a $P(x, \lambda)=\sum_{l=0}^{1} a_{l}(x) \lambda^{l}$, où les $a_{l}$ appartiennent à $\mathcal{H}\left(\Omega_{0}\right)$ et $a_{1}(0) \neq 0$. Pour tout $k \in \mathbb{N}^{\star}$, on a donc

$$
k^{-1} P(x, k)=f\left(x, k^{-1}\right), \quad \text { où } \quad f(x, y)=\sum_{l=0}^{1} a_{l}(x) y^{1-l} ;
$$

la fonction $f$ étant continue au voisinage du compact $\left\{\left(0, k^{-1}\right) ; k \in \mathbb{N}^{\star}\right\} \cup\{(0,0)\}$ et non nulle sur ce compact par hypothèse, il existe un voisinage ouvert $\Omega_{1} \subset \Omega_{0}$ et une constante $c>0$ telle que

$$
\left|f\left(x, k^{-1}\right)\right| \geq c \text { pour tout } x \in \Omega_{1} \text { et tout } k \in \mathbb{N}^{\star} .
$$

D'autre part, on peut supposer

$$
|P(x, 0)| \geq c \text { pour tout } x \in \Omega_{1} .
$$


Par conséquent, on obtient

$$
|P(x, k)| \geq c \max (1, k) \quad \text { pour tout } x \in \Omega_{1} \text { et tout } k \in \mathbb{N},
$$

d'ou le résultat voulu.

$b$. Il est clair que l'opérateur $P: \mathcal{H}\left(\mathcal{R}_{\delta} \times D_{R} \times \Omega\right) \rightarrow \mathcal{H}\left(\mathcal{R}_{\delta} \times D_{R} \times \Omega\right)$ est linéaire continu. Soit $u \in \mathcal{H}\left(\mathcal{R}_{\delta} \times D_{R} \times \Omega\right)$, vu que $\left(t D_{t}\right)^{l} t^{k}=k^{l} t^{k}$ pour tout $k, l \in \mathbb{N}$, on a $P\left(x, t D_{t}\right) u(z, t, x)=\sum_{k \in \mathbb{N}} \frac{t^{k}}{k !} P(x, k) D_{t}^{k} u(z, 0, x)$ dans $\mathcal{R}_{\delta} \times D_{R} \times \Omega$. L'opérateur $P$ est donc injectif d'après $a$; son inverse est formellement donné par la série (2.4). Soient $0<r<s<R$ et $\mathcal{K}$ un compact de $\mathcal{R}_{\delta} \times \Omega$, il existe $c=c(s, \mathcal{K}) \geq 0$ tel que $\left|\frac{t^{k}}{k !} D_{t}^{k} u(z, 0, x)\right| \leq c\left(\frac{r}{s}\right)^{k}$ pour tout $(z, t, x) \in \mathcal{R}_{\delta} \times D_{r} \times \Omega$. Ceci prouve que la série (2.4) converge dans $\mathcal{H}\left(\mathcal{R}_{\delta} \times D_{R} \times \Omega\right)$ puisque $|1 / P(x, k)| \leq 1 / c_{0} ;(2.4)$ étant aussi une série entière de $t$, son image par $P$ est bien égale à $u$. La continuité de $P^{-1}$ résulte directement des majorations précédentes. Enfin, il est clair que $D_{z}^{q} \circ P\left(x, t D_{t}\right)=P\left(x, t D_{t}\right) \circ D_{z}^{q}$, ce qui prouve $b$.

$c$. Ce point se déduit de la démonstration précédente en remarquant que si $K$ est une partie compacte de $\mathcal{O}$, alors il existe un nombre fini d'ouverts $\mathcal{O}_{i}$ relativement compacts dans $\left.D_{R_{i}} \times \Omega_{i},\left(R_{i}, \Omega_{i}\right) \in\right] 0, R^{\prime}[\times \mathcal{U}$, tels que $\mathcal{O}$ soit contenu dans la réunion des $\mathcal{O}_{i}$, d'où le lemme.

Posons $\mathcal{Q}=\{q \in \mathbb{N} \mid 0 \leq q \leq m-1\}$ et pour tout $q \in \mathcal{Q}$, notons $S_{q}$ l'endomorphisme continu de l'espace $\mathcal{H}\left(\mathcal{R}_{\delta} \times D_{R} \times \Omega\right)$ défini par

$$
S_{q}=\left\{\begin{array}{cl}
\sum_{l+|\alpha| \leq 1} a_{l, \alpha}(t, x) t^{1+l} D_{t}^{l} D^{\alpha} \circ P^{-1} & \text { si } q=0, \\
\sum_{0 \leq l \leq 1} t^{l} A_{q}^{l+q}\left(t, x ; D_{t}, D\right) \circ P^{-1} & \text { sinon. }
\end{array}\right.
$$

En remplaçant $u$ par $P^{-1} u$, on en déduit que l'équation (2.3) est équivalente à

$$
u=\mathcal{A} u+v, \quad \text { où } \quad \mathcal{A}=\sum_{q \in \mathcal{Q}} S_{q} D_{z}^{-q}
$$

et que le théorème 1.1 résulte de la proposition suivante.

Proposition 2.4. Soit $\mathcal{O} \subset \mathcal{D}_{0} \times \Omega_{1}$ un voisinage ouvert de l'origine de $\mathbb{C} \times \mathbb{C}^{n}$, il existe $\delta_{0}>0$ et un voisinage ouvert $\mathcal{O}^{\prime} \subset \mathcal{O}$ de l'origine de $\mathbb{C} \times \mathbb{C}^{n}$ tels que : soit $0<\delta \leq \delta_{0}$, pour toute fonction $v \in \mathcal{H}\left(\mathcal{R}_{\delta} \times \mathcal{O}\right)$, l'équation (2.7) admet une unique solution $u \in \mathcal{H}\left(\mathcal{R}_{\delta} \times \mathcal{O}^{\prime}\right)$.

Nous construisons cette solution par la méthode des approximations successives :

$$
u_{0}=v \quad \text { et } \quad u_{k+1}=\mathcal{A} u_{k}+v \quad \text { pour } k \in \mathbb{N} .
$$

Pour étudier la convergence de cette suite $\left(u_{k}\right)$, nous allons la lire sur des chemins tracés sur $\mathcal{R}_{\delta}$. On note $\Gamma_{\delta}$ l'ensemble des chemins $\gamma \in \mathcal{C}^{1}([0, \Lambda] ; \dot{D}(0, \delta))$ d'origine $a_{1}$ paramétrés par leur abscisse curviligne; $\Lambda$ est par conséquent la longueur de $\gamma$. On note enfin $\hat{\gamma}:[0, \Lambda] \rightarrow \mathcal{R}_{\delta}$ le relèvement d'origine $\hat{a}_{1}$ de $\gamma$. Pour toute fonction $u \in \mathcal{H}\left(\mathcal{R}_{\delta} \times \mathcal{O}\right)$ et tout $\gamma \in \Gamma_{\delta}$, on pose

$$
u_{\gamma}(s, t, x)=u(\hat{\gamma}(s), t, x) \text {, où } s \in[0, \Lambda] ;
$$

la fonction $u_{\gamma}$ est continue sur $[0, \Lambda] \times \mathcal{O}$ et holomorphe sur $\mathcal{O}$, autrement dit, $u_{\gamma}$ appartient à l'espace $\mathcal{C}([0, \Lambda] ; \mathcal{H}(\mathcal{O}))$. On observe que

$$
\left(D_{z}^{-1} u\right)_{\gamma}(s, t, x)=\int_{\hat{a}_{1}}^{\hat{\gamma}(s)} u(\sigma, t, x) d \sigma=\int_{0}^{s} u_{\gamma}(\sigma, t, x) \gamma^{\prime}(\sigma) d \sigma
$$


ce qui conduit à poser

$$
\mathcal{D}_{\gamma}^{-1} U(s, t, x) \equiv \int_{0}^{s} U(\sigma, t, x) \gamma^{\prime}(\sigma) d \sigma \quad \text { pour tout } U \in \mathcal{C}([0, \Lambda] ; \mathcal{H}(\mathcal{O})) .
$$

L'opérateur $\mathcal{D}_{\gamma}^{-1}$ ainsi obtenu est clairement un endomorphisme de l'espace $\mathcal{C}([0, \Lambda] ; \mathcal{H}(\mathcal{O}))$. En outre, on a

$$
\left(D_{z}^{q} u\right)_{\gamma}=\mathcal{D}_{\gamma}^{q} u_{\gamma} \quad \text { pour tout entier } q<0 .
$$

Enfin, on a

$$
\left(P^{-1} u\right)_{\gamma}(s, t, x)=P^{-1} u_{\gamma}(s, t, x)
$$

où

$$
P^{-1} U(s, t, x) \equiv \sum_{k \in \mathbb{N}} \frac{t^{k}}{k !} \frac{D_{t}^{k} U(s, 0, x)}{P(x, k)} \quad \text { pour tout } U \in \mathcal{C}([0, \Lambda] ; \mathcal{H}(\mathcal{O})) .
$$

Quitte à reprendre partiellement la démonstration du lemme 2.3, on vérifie aisément le

Lemme 2.5. Soient $\Lambda>0$ et $\mathcal{O}$ un ouvert de la forme (2.5). L'expression (2.9) définit une fonction appartenant à l'espace $\mathcal{C}([0, \Lambda] ; \mathcal{H}(\mathcal{O}))$; de plus, les opérateurs $P^{-1}$ et $\mathcal{D}_{\gamma}^{q}$ commutent quel que soit $q \in \mathbb{Z}_{-}$.

La convergence de la suite $\left(u_{k}\right)$ sera déduite de celle de $\left(u_{k, \gamma}\right)$ grâce au lemme suivant.

Lemme 2.6. Soit $\left(u_{k}\right)$ une suite de $\mathcal{H}\left(\mathcal{R}_{\delta} \times \mathcal{O}\right)$ telle que, pour tout $\gamma \in \Gamma_{\delta}$ de longueur $\Lambda \leq 2 \delta$, la suite $\left(u_{k, \gamma}\right)$ converge uniformément sur tout compact de $[0, \Lambda] \times \mathcal{O}$, alors la suite $\left(u_{k}\right)$ converge uniformément sur tout compact de $\mathcal{R}_{\delta} \times \mathcal{O}$ et $\left(\lim _{k \rightarrow \infty} u_{k}\right)_{\gamma}=\lim _{k \rightarrow \infty} u_{k, \gamma}$.

Démonstration. Étant donné un point $t \in \mathcal{R}_{\delta}$, il est possible de trouver un chemin $\gamma \in \Gamma_{\delta}$ de longueur $\leq 2 \delta$ tel que le relêvement $\hat{\gamma}$ d'origine $\hat{a}_{1}$ de $\hat{\gamma}$ enlace le point $t$; on obtient ainsi la convergence uniforme locale sur $\mathcal{R}_{\delta} \times \mathcal{O}$ et par conséquent $\left(u_{k}\right)$ converge uniformément sur tout compact vers une fonction $u \in \mathcal{H}\left(\mathcal{R}_{\delta} \times \mathcal{O}\right)$. On remarque ensuite que $\sup _{[0, \Lambda] \times K}\left|u_{k, \gamma}-u_{\gamma}\right|=\sup _{\hat{\gamma}([0, \Lambda]) \times K}\left|u_{k}-u\right|$ pour tout $\gamma \in \Gamma_{\delta}$ de longueur $\leq 2 \delta$ et tout compact $K$ de $\mathcal{O}$, d'où le lemme.

Les équations (2.7) et (2.8) lues sur $\gamma$ s'écrivent alors

$$
\left\{\begin{array}{l}
u_{\gamma}=\mathcal{A}_{\gamma} u_{\gamma}+v_{\gamma}, \\
u_{0, \gamma}=0 \text { et } u_{k+1, \gamma}=\mathcal{A}_{\gamma} u_{k, \gamma}+v_{\gamma} \quad \text { pour } k \in \mathbb{N},
\end{array}\right.
$$

où, pour tout $U \in \mathcal{C}([0, \Lambda] ; \mathcal{H}(\mathcal{O}))$,

$$
\mathcal{A}_{\gamma} U=\sum_{q \in \mathcal{Q}} S_{q} \mathcal{D}_{\gamma}^{-q} U
$$

En notant $V=v_{\gamma} \in \mathcal{C}([0, \Lambda] ; \mathcal{H}(\mathcal{O}))$, il s'agit finalement d'étudier l'équation

$$
U=\mathcal{A}_{\gamma} U+V
$$

par la méthode des approximations successives

$$
U_{0}=0 \quad \text { et } \quad U_{k+1}=\mathcal{A}_{\gamma} U_{k}+V \quad \text { pour } k \in \mathbb{N} .
$$

Cette étude repose sur le théorème du point fixe dans un espace de Banach que nous allons préciser. 


\section{Cadre fonctionnel et estimation de L'opérateur $\mathcal{A}_{\gamma}$}

Nous utiliserons les variables $\xi=\sum_{j=1}^{n} x_{j}$, et $\tau=\rho t$, où $\rho$ est un paramètre $>1$.

Définition 3.1. Soit $\phi \in \mathbb{R}_{+}\{\xi\}$ une fonction majorante de rayon de convergence $\geq R>0$, soient $\rho>1, \omega, \Lambda>0$ tels que $\omega \Lambda<R$, on pose

$$
\mathcal{O}(R, \rho, \omega, \Lambda)=\left\{(t, x) \in \mathbb{C} \times \mathbb{C}^{n}|\rho| t\left|+\sum_{j=1}^{n}\right| x_{j} \mid<R-\omega \Lambda\right\}
$$

et on note $\mathcal{K}_{\phi}$ l'espace vectoriel des fonctions $U \in \mathcal{C}([0, \Lambda] ; \mathcal{H}(\mathcal{O}(R, \rho, \omega, \Lambda)))$ pour lesquelles il existe $c \geq 0$ tel que

$$
\forall s \in[0, \Lambda], \quad U(s, t, x) \ll c \phi(\tau+\xi+\omega s) .
$$

Il est clair que $\mathcal{K}_{\phi}$ est un sous espace vectoriel de $\mathcal{C}([0, \Lambda] ; \mathcal{H}(\mathcal{O}(R, \rho, \omega, \Lambda)))$ et que la plus petite constante $c \geq 0$ pour laquelle (3.1) ait lieu est une norme sur cet espace vectoriel, notée $\|\bullet\|_{\phi}$.

Lemme 3.2. L'espace $\mathcal{K}_{\phi}$ est un espace de Banach.

Démonstration. Soit $\left(U_{n}\right)$ une suite de Cauchy de l'espace $\mathcal{K}_{\phi}$ et soit $\varepsilon>0$. Il existe un entier $N \in \mathbb{N}$ tel que pour tout $n, n^{\prime} \geq N$ et tout $s \in[0, \Lambda]$,

$$
\left(U_{n}-U_{n^{\prime}}\right)(s, t, x) \ll \varepsilon \phi(\tau+\xi+\omega s) .
$$

Si $K$ est une partie compacte de $\mathcal{O}(R, \rho, \omega, \Lambda)$, on a donc

$$
\max _{[0, \Lambda] \times K}\left|U_{n}-U_{n^{\prime}}\right| \leq \varepsilon C_{K},
$$

où $C_{K}=\max _{(t, x) \in K} \phi\left(\rho|t|+\sum_{j=1}^{n}\left|x_{j}\right|+\omega \Lambda\right)$ est $<+\infty$ car l'application $(t, x) \mapsto$ $\phi\left(\rho t+\sum_{j=1}^{n} x_{j}+\omega \Lambda\right)$ est holomorphe dans $\mathcal{O}(R, \rho, \omega, \Lambda)$. Ceci prouve que la suite $\left(U_{n}\right)$ est de Cauchy dans l'espace $\mathcal{C}([0, \Lambda] ; \mathcal{H}(\mathcal{O}(R, \rho, \omega, \Lambda)))$, elle converge donc uniformément vers une fonction $U \in \mathcal{C}([0, \Lambda] ; \mathcal{H}(\mathcal{O}(R, \rho, \omega, \Lambda)))$ sur tout compact de $[0, \Lambda] \times \mathcal{O}(R, \rho, \omega, \Lambda)$; a fortiori, pour tout $s \in[0, \Lambda]$ et tout $(h, \alpha) \in \mathbb{N} \times \mathbb{N}^{n}$, la suite $\left(D_{t}^{h} D^{\alpha} U_{n}(s, 0,0)\right)_{n}$ converge vers $D_{t}^{h} D^{\alpha} U(s, 0,0)$. On peut alors passer à la limite sur $n^{\prime}$ dans la relation (3.2) et on en déduit que $\left(U_{n}\right)$ converge vers $U$ dans $\mathcal{K}_{\phi}$.

Lemme 3.3. L'opérateur $\mathcal{D}_{\gamma}^{-1}$ induit une application $\mathcal{D}_{\gamma}^{-1}: \mathcal{K}_{D \phi} \rightarrow \mathcal{K}_{\phi}$ linéaire continue de norme $\leq \omega^{-1}$.

Démonstration. Soit $U \in \mathcal{K}_{D \phi}$; pour tout $s \in[0, \Lambda]$ et tout $(h, \alpha) \in \mathbb{N} \times \mathbb{N}^{n}$, on a

$$
\left|D_{t}^{h} D^{\alpha} U(s, 0,0)\right| \leq\|U\|_{D \phi} \rho^{h} D^{h+|\alpha|+1} \phi(\omega s),
$$

d'où

$$
\left|D_{t}^{h} D^{\alpha} \mathcal{D}_{\gamma}^{-1} U(s, 0,0)\right| \leq\|U\|_{D \phi} \rho^{h} \int_{0}^{s} D^{h+|\alpha|+1} \phi(\omega \sigma) d \sigma \leq\|U\|_{D \phi} \frac{\rho^{h}}{\omega} D^{h+|\alpha|} \phi(\omega s) ;
$$

c'est-à-dire $\mathcal{D}_{\gamma}^{-1} U \ll \omega^{-1}\|U\|_{D \phi} \phi(\tau+\xi+\omega s)$, ce qui prouve le lemme. 
Dans tout ce qui suit, $\phi \in \mathbb{R}_{+}\{\xi\}$ désignera une fonction majorante de rayon de convergence $\geq R>0$ vérifiant $0 \ll(R-\xi) \phi(\xi)$.

Rappelons [4, Proposition 6.1] que, si $\phi \in \mathbb{R}_{+}[[\xi]]$ vérifie $0 \ll(R-\xi) \phi(\xi)$, alors les dérivées $D^{p} \phi$ de $\phi$ vérifient également $0 \ll(R-\xi) D^{p} \phi(\xi)$ et

$$
\frac{\eta R}{\eta R-\xi} D^{p} \phi(\xi) \ll \frac{\eta}{\eta-1} D^{p} \phi(\xi) \text { pour tout } \eta>1 \text { et tout } p \in \mathbb{N} .
$$

Pour tout $R>0$, on pose

$$
\Delta_{R}=\left\{x \in \mathbb{C}^{n}\left|\max _{1 \leq j \leq n}\right| x_{j} \mid<R\right\} .
$$

On fixe une fois pour toutes $\eta>1$ et $R_{0}>0$ tels que $\mathcal{D}_{0} \times \Omega_{1}\left(\subset \mathcal{D}_{0} \times \Omega_{0}=\mathcal{O}_{0}\right)$ contienne le polydisque $\bar{D}_{\eta R_{0}} \times \bar{\Delta}_{\eta R_{0}}$. D'après le lemme 2.3- $a$ et les inégalités de Cauchy, on a

$$
\left.\left.\frac{1}{P(x, k)} \ll \frac{c_{0}^{-1}}{k+1} \frac{\eta R}{\eta R-\xi} \text { pour tout } R \in\right] 0, R_{0}\right] \text { et tout } k \in \mathbb{N} .
$$

Il est clair que $\mathcal{O}(R, \rho, \omega, \Lambda) \subset D_{R} \times \Delta_{R} \subset \mathcal{D}_{0} \times \Omega_{1}$. En outre, en posant pour tout $R>0$,

$$
\Omega_{R}=\left\{x \in \mathbb{C}^{n}\left|\sum_{j=1}^{n}\right| x_{j} \mid<R\right\}
$$

on observe que

$$
\mathcal{O}(R, \rho, \omega, \Lambda)=\bigcup_{r \in] 0, R-\omega \Lambda[} D_{r / \rho} \times \Omega_{R-\omega \Lambda-r}
$$

autrement dit, $\mathcal{O}(R, \rho, \omega, \Lambda)$ est un ouvert de la forme (2.5). Voici alors une estimation de $P^{-1}$.

Lemme 3.4. Il existe une constante $c\left(=c_{0}^{-1} \frac{\eta}{\eta-1}\right)>0$ telle que $:$ soit $\left.\left.R \in\right] 0, R_{0}\right]$, si $U \in \mathcal{K}_{\phi}$, alors $P^{-1} U \in \mathcal{C}([0, \Lambda] ; \mathcal{H}(\mathcal{O}(R, \rho, \omega, \Lambda))$ vérifie

$$
P^{-1} U \ll c\|U\|_{\phi} \sum_{k=0}^{\infty} \tau^{k} \frac{D^{k} \phi(\xi+\omega s)}{(k+1) !} .
$$

Démonstration. Soit $U \in \mathcal{K}_{\phi}$, alors d'après (3.5) et le lemme 2.5-c, $P^{-1} U \in$ $\mathcal{C}([0, \Lambda] ; \mathcal{H}(\mathcal{O}(R, \rho, \omega, \Lambda)))$. La relation $U(s, t, x) \ll\|U\|_{\phi} \phi(\tau+\xi+\omega s)$ signifie aussi

$$
D_{t}^{k} U(s, 0, x) \ll\|U\|_{\phi} \rho^{k} D^{k} \phi(\xi+\omega s) \quad \text { pour tout } k \in \mathbb{N} .
$$

$\mathrm{Vu}(2.9)$, on a $D_{t}^{k}\left(P^{-1} U\right)(s, 0, x)=\frac{D_{t}^{k} U(s, 0, x)}{P(x, k)}$ et, d'après $(3.3)$ et $(3.4)$, on en déduit

$$
\frac{1}{k !} D_{t}^{k}\left(P^{-1} U\right)(s, 0, x) \ll c\|U\|_{\phi} \rho^{k} \frac{D^{k} \phi(\xi+\omega s)}{k !(k+1)} \text { pour tout } k \in \mathbb{N},
$$

c'est-à-dire le résultat voulu.

Note. L'identité

$$
\sum_{k=0}^{\infty} \tau^{k} \frac{D^{k} \phi(\xi)}{(k+1) !}=\left(t D_{t}+1\right)^{-1}[\phi(\tau+\xi)]
$$

montre que l'opérateur $P^{-1}$ se majore comme l'opérateur $\left(t D_{t}+1\right)^{-1}$. 
Nous allons maintenant contrôler les opérateurs $S_{q}$. A cet effet, nous utiliserons le lemme suivant [10, lemme 1.4].

Lemme 3.5. Soit $\phi \in \mathbb{R}_{+}[[\xi]]$ tel que $0 \ll(R-\xi) \phi(\xi)$, alors pour tout entier $i \leq j$,

$$
R^{i} \frac{D^{i} \phi}{i !} \ll R^{j} \frac{D^{j} \phi}{j !} .
$$

Dans les majorations qui vont suivre, toute constante qui dépend des paramètres $c_{0}, \eta, R_{0}$ déjà fixés, sera notée $c$, sauf mention expresse. Étant donné que $\bar{D}_{\eta R_{0}} \times$ $\bar{\Delta}_{\eta R_{0}} \subset \mathcal{O}_{0}$, tous les coefficients des opérateurs $S_{q}$ sont holomorphes et bornés sur $D_{\eta R_{0}} \times \Delta_{\eta R_{0}}$. Soit $\left.\left.R \in\right] 0, R_{0}\right]$, si $b$ désigne l'un de ces coefficients, on a d'après les inégalités de Cauchy,

$$
b(t, x) \ll c \frac{\eta R}{\eta R-(\tau+\xi)} .
$$

Lemme 3.6. Il existe $c>0$ tel que, pour tout $\left.R \in] 0, R_{0}\right]$, l'opérateur $S_{q}$ induise une application linéaire continue $S_{q}: \mathcal{K}_{\phi} \rightarrow \mathcal{K}_{D^{a} \phi}$ de norme

$$
\left\|S_{q}\right\| \leq \begin{cases}c \rho^{-1} & \text { si } q=0 \\ c \rho^{q} & \text { si } q>0 .\end{cases}
$$

Démonstration. Soit $U \in \mathcal{K}_{\phi} ;$ d'après le lemme 2.5, $S_{q} U \in \mathcal{C}([0, \Lambda]$; $\mathcal{H}(\mathcal{O}(R, \rho, \omega, \Lambda)))$. Si $q=0, S_{q}$ est une somme finie de termes de la forme

$$
b(t, x) t^{l+1} D_{t}^{l} D^{\alpha} P^{-1}, \quad \text { où } \quad l+|\alpha| \leq 1 .
$$

Grâce à (3.3) et (3.7), nous pouvons faire abstraction du coefficient $b(t, x)$. Vu (3.6), on a

$$
t^{l+1} D_{t}^{l} D^{\alpha} P^{-1} U \ll c\|U\|_{\phi} \rho^{-1} \sum_{k \geq l} \tau^{k+1} \frac{k !}{(k-l) !} \frac{D^{k+|\alpha|} \phi(\xi+\omega s)}{(k+1) !} .
$$

Étant donné que $|\alpha| \leq 1$, on a d'après le lemme 3.5,

$$
\frac{D^{k+|\alpha|} \phi}{(k+|\alpha|) !} \ll R^{1-|\alpha|} \frac{D^{k+1} \phi}{(k+1) !} .
$$

On note que $R^{1-|\alpha|} \leq R_{0}^{1-|\alpha|} \leq c$; on remarque ensuite que

$$
\frac{k !}{(k-l) !} \frac{(k+|\alpha|) !}{(k+1) !} \leq 1 \quad \text { pour tout } k \geq l
$$

car $l+|\alpha| \leq 1$. Il en résulte que

$$
t^{l+1} D_{t}^{l} D^{\alpha} P^{-1} U \ll c\|U\|_{\phi} \rho^{-1} \sum_{k \geq l} \tau^{k+1} \frac{D^{k+1} \phi(\xi+\omega s)}{(k+1) !},
$$

d'où le résultat voulu dans ce cas.

Lorsque $q$ est $>0$, rappelons que l'ordre des opérateurs $A_{q}^{l+q}$ est $\leq l+q$; à nouveau, on en déduit qu'il s'agit de majorer les opérateurs

$$
t^{l} D_{t}^{h} D^{\alpha} P^{-1}, \quad \text { où } \quad h+|\alpha| \leq l+q, \quad 0 \leq l \leq 1 .
$$

$\mathrm{Vu}(3.6)$, on a

$$
t^{l} D_{t}^{h} D^{\alpha} P^{-1} U \ll c\|U\|_{\phi} \rho^{h-l} \sum_{k \geq h} \tau^{k-h+l} \frac{k !}{(k-h) !} \frac{D^{k+|\alpha|} \phi(\xi+\omega s)}{(k+1) !} .
$$


Étant donné que $h+|\alpha| \leq l+q$, on a $\rho^{h-l} \leq \rho^{q}$ et, d'après le lemme 3.5,

$$
D^{k+|\alpha|} \phi \ll c D^{k-h+l+q} \phi, \quad \text { où } \quad c=R_{0}^{l+q-(h+|\alpha|)} .
$$

On observe alors que

$$
\frac{k !}{(k-h) !} \frac{(k-h+l) !}{(k+1) !} \leq 1 \quad \text { pour tout } k \geq h
$$

car $0 \leq l \leq 1$. On obtient donc

$$
t^{l} D_{t}^{h} D^{\alpha} P^{-1} U \ll c\|U\|_{\phi} \rho^{q} \sum_{k \geq h} \tau^{k-h+l} \frac{D^{k-h+l+q} \phi(\xi+\omega s)}{(k-h+l) !},
$$

d'où le lemme.

Les lemmes 2.5 et 3.3 permettent d'en déduire aisément le corollaire suivant.

Lemme 3.7. Il existe $c>0$ tel que, pour tout $\left.R \in] 0, R_{0}\right]$, l'opérateur $\mathcal{A}_{\gamma}$ induise un endomorphisme continu de l'espace $\mathcal{K}_{\phi}$ de norme $\leq c\left[\rho^{-1}+\sum_{q \in \mathcal{Q}^{\star}}\left(\omega^{-1} \rho\right)^{q}\right]$.

\section{Preuve de la proposition 2.4}

En choisissant $\rho \geq 1$ tel que $c \rho^{-1}<1 / 2$ et en prenant ensuite $\omega>0$ suffisamment grand pour que $c \sum_{q \in \mathcal{Q}^{\star}}\left(\omega^{-1} \rho\right)^{q}<1 / 2$, on obtient $\left\|\mathcal{A}_{\gamma}\right\|<1$.

Soit $\mathcal{O} \subset \mathcal{D}_{0} \times \Omega_{1}$ un voisinage ouvert de l'origine de $\mathbb{C} \times \mathbb{C}^{n}$. On choisit $\left.\left.R \in\right] 0, R_{0}\right]$ tel que $\bar{D}_{R} \times \bar{\Delta}_{R} \subset \mathcal{O}$ puis $\Lambda_{0}>0$ tel que $\omega \Lambda_{0}<R$; on pose $\delta_{0}=\Lambda_{0} / 2$ et $\mathcal{O}^{\prime}=\mathcal{O}\left(R, \rho, \omega, \Lambda_{0}\right)$. Soient $0<\delta \leq \delta_{0}$ et $v \in \mathcal{H}\left(\mathcal{R}_{\delta} \times \mathcal{O}\right)$. Si $\gamma \in \Gamma_{\delta}$ est un chemin de longueur $\Lambda \leq 2 \delta$, on pose $V=v_{\gamma} \in \mathcal{C}([0, \Lambda] ; \mathcal{H}(\mathcal{O})) ;$ l'ensemble $\hat{\gamma}([0, \Lambda]) \times \bar{D}_{R} \times \bar{\Delta}_{R}$ étant une partie compacte de $\mathcal{R}_{\delta} \times \mathcal{O}$, il existe une constante $c_{\gamma} \geq 0$ telle que $V$ soit bornée par $c_{\gamma}$ sur $[0, \Lambda] \times D_{R} \times \Delta_{R}$. D'après les inégalités de Cauchy, on a donc

$$
\forall s \in[0, \Lambda], \quad V(s, t, x) \ll c_{\gamma} R \phi(\tau+\xi),
$$

où $\phi$ désigne la simple fonction majorante

$$
\phi(\xi)=\frac{1}{R-\xi}
$$

à laquelle on peut appliquer les résultats du paragraphe pécédent. Étant donné que $\omega \Lambda<R$, on a

$$
\forall s \in[0, \Lambda], \quad \phi(\tau+\xi) \ll \sum_{k=0}^{+\infty} \frac{(\omega s)^{k}}{k !} D^{k} \phi(\tau+\xi)=\phi(\tau+\xi+\omega s)
$$

et on en déduit que la fonction $V$ appartient à l'espace $\mathcal{K}_{\phi}$. Il en résulte que l'équation (2.12) admet une unique solution $\mathcal{U}$ appartenant à $\mathcal{K}_{\phi}$ et que la relation (2.13) définit une suite $\left(U_{k}\right)$ de $\mathcal{K}_{\phi}$ qui converge vers $U$ dans $\mathcal{K}_{\phi}$. Vu que $\Lambda \leq \Lambda_{0}$, on observe que $\mathcal{O}^{\prime} \subset \mathcal{O}(R, \rho, \omega, \Lambda)$ et, comme expliqué dans la preuve du lemme 3.2, il existe pour tout compact $K$ de $\mathcal{O}^{\prime}$ une constante $C_{K}$ telle que

$$
\forall U \in \mathcal{K}_{\phi}, \quad \max _{[0, \Lambda] \times K}|U| \leq C_{K}\|U\|_{\phi}
$$

Par conséquent, la suite $\left(U_{k}\right)$ converge vers $U$ dans l'espace $\mathcal{C}\left([0, \Lambda] ; \mathcal{H}\left(\mathcal{O}^{\prime}\right)\right)$. Si $\left(u_{k}\right)$ est la suite de $\mathcal{H}\left(\mathcal{R}_{\delta} \times \mathcal{O}^{\prime}\right)$ définie par (2.8), il est clair d'après le paragraphe 2 que $u_{k, \gamma}=U_{k}$; d'après le lemme 2.6, la suite $\left(u_{k}\right)$ converge donc uniformément sur tout compact de $\mathcal{R}_{\delta} \times \mathcal{O}^{\prime}$ vers une fonction $u \in \mathcal{H}\left(\mathcal{R}_{\delta} \times \mathcal{O}^{\prime}\right)$. En outre, l'ouvert $\mathcal{O}^{\prime}$ étant de la forme (2.5) vu (3.5), l'opérateur $\mathcal{A}: \mathcal{H}\left(\mathcal{R}_{\delta} \times \mathcal{O}^{\prime}\right) \rightarrow \mathcal{H}\left(\mathcal{R}_{\delta} \times \mathcal{O}^{\prime}\right)$ est continu 
par composition d'après les lemmes 2.2 et 2.3. On en déduit que $u$ vérifie (2.7); montrons enfin que cette solution est unique. Si $u \in \mathcal{H}\left(\mathcal{R}_{\delta} \times \mathcal{O}^{\prime}\right)$ vérifie $u=\mathcal{A} u$, on choisit $\left.\left.R^{\prime} \in\right] 0, R_{0}\right]$ et $\left.\left.\Lambda^{\prime} \in\right] 0,2 \delta\right]$ tels que $\bar{D}_{R^{\prime}} \times \bar{\Delta}_{R^{\prime}} \subset \mathcal{O}^{\prime}$ et $\omega \Lambda^{\prime}<R^{\prime}$; on pose $\delta^{\prime}=\Lambda^{\prime} / 2$ et $\mathcal{O}^{\prime \prime}=\mathcal{O}\left(R^{\prime}, \rho, \omega, \Lambda^{\prime}\right)$. Si $\gamma \in \Gamma_{\delta^{\prime}}$ est un chemin de longueur $\Lambda \leq \Lambda^{\prime}$, il s'en suit comme précédemment que $u_{\gamma}$ appartient à l'espace $\mathcal{K}_{\phi}$ associé à ces nouveaux paramètres. Vu que $u_{\gamma}=\mathcal{A}_{\gamma} u_{\gamma}$ et $\left\|\mathcal{A}_{\gamma}\right\|<1$, on a $u_{\gamma}=0$ sur $[0, \Lambda] \times \mathcal{O}\left(R^{\prime}, \rho, \omega, \Lambda\right) \supset[0, \Lambda] \times \mathcal{O}^{\prime \prime} ;$ ce chemin $\gamma$ étant arbitraire, on a $u=0$ sur $\mathcal{R}_{\delta^{\prime}} \times \mathcal{O}^{\prime \prime}$, d'où $u \equiv 0$. Ceci termine la preuve de la proposition 2.4.

\section{RÉFÉRENCES}

1. M. S. Baouendi et C. Goulaouic, Cauchy problems with characteristic initial hypersurface, Comm. Pure Appl. Math., 26, 1973, pp. 455-475. MR0338532 (49:3296)

2. S. Fujiié, Représentation hypergéométrique des singularités de la solution du problème de Cauchy caractéristique à données holomorphes, Comm. Partial Diff. Eq., 18 (9-10), 1993, pp. 1589-1629. MR1239925 (95f:35005)

3. S. Fujiié, Solutions ramifiées des problèmes de Cauchy caractéristiques et fonctions hypergéométriques à deux variables, Tohoku Mathematical Publications. 6. Sendai : Tohoku University, 59 pp., Thèse, 1997. MR1478161(99b:33030)

4. Y. Hamada, J. Leray et C. Wagschal, Systèmes d'équations aux dérivées partielles à caractéristiques multiples : problème de Cauchy ramifié; hyperbolicité partielle, J. Math. Pures Appl., 55, 1976, pp. 297-352. MR0435614 (55:8572)

5. J. Leray, Problème de Cauchy. I. Uniformisation de la solution du problème linéaire analytique de Cauchy près de la variété qui porte les données de Cauchy, Bull. Soc. Math. France, 85, 1957, pp. 389-429. MR0103328 (21:2102)

6. A. Nabaji et C. Wagschal, Singularités à croissance lente, J. Math. Pures Appl., 72, 1993, pp. 335-375. MR1228997 (94k:35007)

7. S. Ouchi, Singularities of solutions of equations with noninvolutive characteristics. I. The case of second order Fuchsian equations, J. Math. Soc. Japan, 45 (2), 1993, pp. 215-251. MR.1206651 (94h:35006)

8. P. Pongérard et C. Wagschal, Ramification non abélienne, J. Math. Pures Appl., 77, 1998, pp. 51-88. MR.1617590 (99d:35010)

9. P. Pongérard, Sur une classe d'équations de Fuchs non linéaires, J. Math. Sci. Univ. Tokyo, 7, 2000, pp. 423-448. MR1792735 (2001j:35003)

10. P. Pongérard, Problème de Cauchy caractéristique à solution entière, J. Math. Sci. Univ. Tokyo, 8, 2001, pp. 89-105. MR.1818907 (2001m:35009)

11. P. Pongérard, Ramification des solutions du problème de Cauchy fuchsien au voisinage de l'hypersurface initiale, J. Math. Sci. Univ. Tokyo, 12, 2005, pp. 493-512. MR2206356 (2007b:35012)

12. J. Urabe, Meromorphic representations of the solutions of the singular Cauchy problem. II, J. Math. Kyoto Univ., 28 (2), 1988, pp. 335-342. MR953181 (92c:35005)

13. C. Wagschal, Sur le problème de Cauchy ramifié, J. Math. Pures Appl., 53, 1974, pp. 147-164. MR.0382832 (52:3714)

14. H. Yamane, Singularities in Fuchsian Cauchy problems with holomorphic data, Publ. RIMS, Kyoto Univ., 34, 1998, pp. 179-190. MR.1617067 (99h:35005)

Université de La Réunion, 23 allée des rubis, 97400 Saint-Denis, La Réunion, France

E-mail address: marc-patrice.pongerard@univ-reunion.fr 